\title{
Italian doctors call for protecting healthcare workers and boosting community surveillance during covid-19 outbreak
}

\author{
Filippo Anelli president ${ }^{1}$, Giovanni Leoni vice president ${ }^{1}$, Roberto Monaco secretary general ${ }^{1}$, \\ Cosimo Nume president ${ }^{2}$, Roberto Carlo Rossi president ${ }^{3}$, Guido Marinoni president ${ }^{4}$, Gianluigi \\ Spata president ${ }^{5}$, Donato De Giorgi president ${ }^{6}$, Luigi Peccarisi vice president ${ }^{6}$, Alessandro Miani \\ president ${ }^{7}$, Ernesto Burgio director of Scientific Committee ${ }^{7}$, Ivan Gentile director of Infectious \\ Disease Division ${ }^{8}$, Annamaria Colao chair holder ${ }^{8}$, Maria Triassi full professor of public health ${ }^{8}$, \\ Prisco Piscitelli scientific officer ${ }^{78}$
}

\begin{abstract}
${ }^{1}$ Italian Federation of Medical Professional Associations (FNOMCEO), Via Ferdinando di Savoia 1, 00196 Rome, Italy; ${ }^{2}$ Medical Professional Association (OMCEO) of Taranto, Via F Crispi 107, 74123, Taranto, Italy; ${ }^{3}$ Medical Professional Association (OMCEO) of Milan, Via Lanzone 31 , 20123 Milan, Italy; ${ }^{4}$ Medical Professional Association (OMCEO) of Bergamo, Via Giacomo Manzu' 25, 24122 Bergamo, Italy; ${ }^{5}$ Medical Professional Association (OMCEO) of Como, Viale Massenzio Masia 30, 22100 Como, Italy; ${ }^{6}$ Medical Professional Association (OMCEO) of Lecce, Via Nazario Saur, 31, 73100, Lecce, Italy; ${ }^{7}$ Italian Society of Environmental Medicine (SIMA), Via Monte Leone 2, 20149 Milano; ${ }^{8}$ UNESCO Chair on Health Education and Sustainable Development, Federico II University, Via S Pansini, 5, 80131, Naples Italy
\end{abstract}

Italy is in a dramatic situation owing to the spread of covid- $19,{ }^{1}$ but we could do more to protect medical doctors and all hospital workers, including nurses, therapists, technicians, and support staff. Beyond the personal risks that doctors and healthcare workers are facing directly-highlighted by the death of general practitioner and national delegate for continuous medical education Roberto Stella in Varese-hospitals and medical personnel are a potential vehicle for spread of covid-19. ${ }^{2}$

The New England Journal of Medicine has reported on the huge proportion of infected people who remain asymptomatic and their role in spreading the epidemic. ${ }^{2}$ At the same time, as pointed out by Tedros Ghebreyesus, director general of the World Health Organization, about $41 \%$ of covid-19 cases confirmed in Wuhan resulted from hospital related transmission. ${ }^{3}$

A hospital-centric model has been shown to be inadequate in coping with the coronavirus outbreak. Epidemics must be counteracted through well planned community surveillance at the local level, by identifying and isolating at home suspected or symptomatic cases. This has become evident as entire hospitals in Italy have closed because of the infection circulating among doctors and nurses. By 22 March, 4824 healthcare workers had been infected ( $9 \%$ of total cases), with 24 doctors dead-these figures are worse than those observed in China (3300 healthcare workers infected, and 23 doctors dead).
Protecting healthcare workers is a crucial factor both for control of the outbreak and for continuing to provide necessary care to people with covid-19 and to all the other patients who need treatment at home or in hospital. ${ }^{4}$

Along with adequate urgent supplies of respiratory protective devices and disposable gowns, which are unacceptably still lacking in the middle of the outbreaks, we propose providing at least all symptomatic healthcare workers with validated rapid response tests, registered at the Italian Ministry of Health, which have $100 \%$ specificity (ability to detect negative cases) and produce a result in 15 to 45 minutes, depending on the different products.

These rapid response tests should be systematically provided at least to healthcare workers with any potential symptoms of covid-19 (even mild and in absence of fever) and to those known to have been in contact with suspected or confirmed cases. Then healthcare services would be sure that personnel with negative results could start working in hospitals, ambulances, or home based and long term care facilities for elderly people and critically ill patients. Rapid response tests performed on healthcare workers should be confirmed by pharyngeal swabs (twice in the same week) and tested with the most reliable PCR methods, the results of which are usually delivered within 48 hours. 


\section{Competing interests: None declared.}

Paterlini M. On the front lines of coronavirus: the Italian response to covid-19. BMJ 2020;368:m1065. 10.1136/bmj.m1065 32179517

2 Rosenbaum L. Facing covid-19 in Italy - ethics, logistics, and therapeutics on the epidemic's front line. N Engl J Med 2020; 10.1056/NEJMp2005492. 32187459

3 Wu Z, McGoogan JM. Characteristics of and important lessons from the coronavirus disease 2019 (covid-19) outbreak in China: summary of a report of 72314 cases from the Chinese Center for Disease Control and Prevention. JAMA 2020;

10.1001/jama.2020.2648. 32091533 4 Chang D, Xu H, Rebaza A, Sharma L, Dela Cruz CS. Protecting healthcare workers from

10.1016/S2213-2600(20)30066-7. 32061333

Published by the BMJ Publishing Group Limited. For permission to use (where not already granted under a licence) please go to http://group.bmj.com/group/rights-licensing/ permissions 